\title{
Delayed Feedback Control of Hidden Chaos in the Unified Chaotic System between the Sprott C System and Yang System
}

\author{
Huijian Zhu and Lijie Li $\mathbb{C}$ \\ Guangxi Colleges and Universities Key Laboratory of Complex System Optimization and Big Data Processing, \\ Yulin Normal University, Yulin 537000, Guangxi, China
}

Correspondence should be addressed to Lijie Li; lijieguangxi@126.com

Received 8 October 2021; Accepted 1 November 2021; Published 19 November 2021

Academic Editor: Viet-Thanh Pham

Copyright (c) 2021 Huijian Zhu and Lijie Li. This is an open access article distributed under the Creative Commons Attribution License, which permits unrestricted use, distribution, and reproduction in any medium, provided the original work is properly cited.

\begin{abstract}
In this paper, the influence of delayed feedback on the unified chaotic system from the Sprott $\mathrm{C}$ system and Yang system is studied. The Hopf bifurcation and dynamic behavior of the system are fully studied by using the central manifold theorem and bifurcation theory. The explicit formula, bifurcation direction, and stability of the periodic solution of bifurcation are given correspondingly. The Hopf bifurcation diagram and chaotic phenomenon are also analyzed by numerical simulation to prove the correctness of the theory. It shows that this delay control can only be applied to the hidden chaos with two stable equilibria.
\end{abstract}

\section{Introduction}

Since Lorentz inadvertently discovered chaos in a threedimensional autonomous system [1] in 1963, more and more scholars began to study the chaos of various systems. Sprott [2-4] found nineteen simple chaotic systems, which may have no equilibra, one equilibrium, or two equilibra. Some classical three-dimensional autonomous chaotic systems, such as Lorentz system [1] and Chen system [5], have a saddle point and two unstable saddle foci. Other three-dimensional chaotic systems [6] have two unstable saddle foci. A chaotic system with one saddle and two stable node foci was discovered by Yang and Chen [7]. Some current studies on these systems including theoretical proof and numerical simulation can be found in the literature [8-19].

In 2000, Yang, Wei, and Chen [20] introduced a new three-dimensional chaotic system that is very similar to Lorentz system and Chen system, but it has only two stable node foci. The related types of chaotic systems have been analyzed and numerically studied in detail [21-24]. It has become very important to study the local and global properties of systems with chaotic phenomena. As it is known, hidden attractors are known as the kind of attractors whose domain of attraction is outside the equilibrium points; meanwhile, the domain of attraction in self-excited attractors is related to the unstable equilibrium points [25-27]. Finding hidden attractors in complex variable chaotic systems is even more difficult than finding their real variable counterparts. In recent years, an increasing number of scholars have paid attention to the control and utilization of chaos [28-31] for stabilizing the system with chaotic behaviors. It is also worth noting that theories and methods of controlling hidden chaos in continuous dynamical systems have been developed. This paper mainly studies the following unified chaotic system from $[2,20,24]$ :

$$
\left\{\begin{array}{l}
\dot{x}=a(y-x) \\
\dot{y}=-c y-x z+k(y(t-\tau)-y), \\
\dot{z}=-b+k_{1} x y+k_{2} y^{2},
\end{array}\right.
$$

where $a, b$, and $c$ are the positive real parameters, $k, k_{1}$, and $k_{2}$ are the control parameters, and $\tau$ is the delay parameter. For parameter values $\left(a, b, c, k, k_{1}, k_{2}\right)=(1,1,0,0,0,1)$, system (1) becomes the Sprott C system. For parameter values $\left(k, k_{1}, k_{2}\right)=(0,1,0)$, system (1) is a general expression for the Yang system. Moreover, for parameter values $\left(a, b, c, k, k_{1}, k_{2}\right)=$ $(10,100,9.8,0,0.08,0.01)$, system (1) without delay has two 
stable equilibria, whose three characteristic values are $\lambda_{1}=$ -19.3894 and $\lambda_{2,3}=-0.2053 \pm 10.1542 i$. Figures $1(\mathrm{a})$ and 1(b), respectively, show time series and the projection of chaotic attractors on the $y-z$ plane. Therefore, system (1) has a hidden attractor coexisting with two stable node foci for initial values $(0.98,-1.82,0.49)$. Therefore, we want to consider the stability of system (1) with direct delay feedback from theoretical analysis and numerical study. At the same time, when all equilibria are stable, we want to show the results that system (1) will turn to chaotic attractor from Hopf bifurcation [32].

The organization of this paper is as follows: In Section 2, the bifurcation conditions of Hopf bifurcation in delayed system (1) are discussed. In Section 3, based on the central manifold theorem and bifurcation theory, the direction and stability of Hopf bifurcation are analyzed in detail. In Section 4, numerical simulations illustrate our theoretical results. Finally, the conclusion is given in Section 5.

\section{Existence of Hopf Bifurcation in System (1)}

If $k_{1}+k_{2}>0$, system (1) possesses two equilibria $E_{1,2}$ $\left( \pm \sqrt{b} / \sqrt{k_{1}+k_{2}}, \pm \sqrt{b} / \sqrt{k_{1}+k_{2}},-c\right)$. Because of the symmetry of $E_{1}$ and $E_{2}$, it is sufficient to analyze the properties of only one of them. So, the rest of the discussion is going to be about $E_{1}$. By the following linear transformation to shift $E_{1}$ to the origin,

$$
\begin{aligned}
& x_{1}=x-\frac{\sqrt{b}}{\sqrt{k_{1}+k_{2}}}, \\
& y_{1}=y-\frac{\sqrt{b}}{\sqrt{k_{1}+k_{2}}}, \\
& z_{1}=x+c,
\end{aligned}
$$

the controlled system (1) is

$$
\left\{\begin{array}{l}
\dot{x}_{1}=a\left(y_{1}-x_{1}\right), \\
\dot{y}_{1}=c\left(x_{1}-y_{1}\right)-\frac{\sqrt{b}}{\sqrt{k_{1}+k_{2}}} z_{1}-x_{1} z_{1}+k\left[y_{1}(t-\tau)-y_{1}\right] \\
\dot{z}_{1}=\frac{\sqrt{b} k_{1}}{\sqrt{k_{1}+k_{2}}} x_{1}+\frac{\sqrt{b}\left(k_{1}+2 k_{2}\right)}{\sqrt{k_{1}+k_{2}}} y_{1}+k_{1} x_{1} y_{1}+k_{2} y_{1}^{2}
\end{array}\right.
$$

The characteristic equation corresponding to the linear matrix of equation (3) is

$$
\begin{gathered}
\lambda^{3}+(a+c+k) \lambda^{2}+\left(\frac{k_{1}+2 k_{2}}{k_{1}+k_{2}} b+a k\right) \lambda+2 a b \\
-\left(\lambda^{2}+a \lambda\right) k e^{-\lambda \tau}=0 .
\end{gathered}
$$

When $\tau=0$, equation (4) becomes

$$
\lambda^{3}+(a+c) \lambda^{2}+\frac{k_{1}+2 k_{2}}{k_{1}+k_{2}} b \lambda+2 a b=0 .
$$

According to the Routh-Hurwitz criterion, in equation (5), under the following conditions, there are three roots in the negative real part:

$$
k_{2}>\max \left\{-k_{1},-\frac{1}{2}(c-a) k_{1}\right\} .
$$

The classification conditions of reference equilibrium point, $E_{1}$, and $E_{2}$ are local stable nodes or focal points.

For the sake of analysis, let us reduce equation (4) to

$$
\lambda^{3}+a_{2} \lambda^{2}+a_{1} \lambda+a_{0}+\left(b_{2} \lambda^{2}+b_{1} \lambda\right) e^{-\lambda \tau}=0
$$

where $a_{2}=a+c+k, a_{1}=\left(k_{1}+2 k_{2}\right) / \quad\left(k_{1}+k_{2}\right) b+a k, a_{0}$ $=2 a b, b_{2}=-k$, and $b_{1}=-a k$.

Since Hopf bifurcations must have a pair of pure imaginary roots at the system equilibrium point, we might as well establish system (1) having a pair of pure imaginary roots, that is, $i \rho$, so we can substitute the roots into equation (4):

$$
\begin{gathered}
-\rho^{3} i-a_{2} \rho^{2}+a_{1} \rho i+a_{0}+\left(-b_{2} \rho^{2}+b_{1} \rho i\right) \\
\cdot[\cos (\rho \tau)-i \sin (\rho \tau)]=0,
\end{gathered}
$$

where we separate the real part from the imaginary part:

$$
\left\{\begin{array}{l}
\rho^{3}-a_{1} \rho=b_{2} \rho^{2} \sin (\rho \tau)+b_{1} \rho \cos (\rho \tau), \\
a_{2} \rho^{2}-a_{0}=b_{1} \rho \sin (\rho \tau)-b_{2} \rho^{2} \cos (\rho \tau),
\end{array}\right.
$$

which leads to

$$
\rho^{6}+\left(a_{2}^{2}-b_{2}^{2}-2 a_{1}\right) \rho^{4}+\left(a_{1}^{2}-2 a_{0} a_{2}-b_{1}^{2}\right) \rho^{2}+a_{0}^{2}=0 .
$$

Let $s=\rho^{2}$ and let us denote $p=a_{2}^{2}-b_{2}^{2}-2 a_{1}, q=a_{1}^{2}-2 a_{0} a_{2}-b_{1}^{2}$, and $r=a_{0}^{2}$; then, equation (10) becomes

$$
s^{3}+p s^{2}+q s+r=0 .
$$

Let

$$
h(s)=s^{3}+p s^{2}+q s+r .
$$

From equation (11), we have

$$
h^{\prime}(s)=3 s^{2}+2 p s+q .
$$

Denote $\Delta=p^{2}-3 q$. When $\Delta>0$ and $r>0$, we can solve for two real roots of equation $h^{\prime}(s)=0$ as follows:

$$
\begin{aligned}
& s_{1}^{*}=\frac{-p+\sqrt{\Delta}}{3}, \\
& s_{2}^{*}=\frac{-p-\sqrt{\Delta}}{3} .
\end{aligned}
$$

Noticing that $r=a_{0}^{2}>0$ and $\lim _{s \longrightarrow+\infty} h(s)=+\infty$, we can get results similar to [9].

Lemma 1. The following results hold:

(1) Equation (11) does not have positive real roots if $\Delta=p^{2}-3 q \leq 0$ 


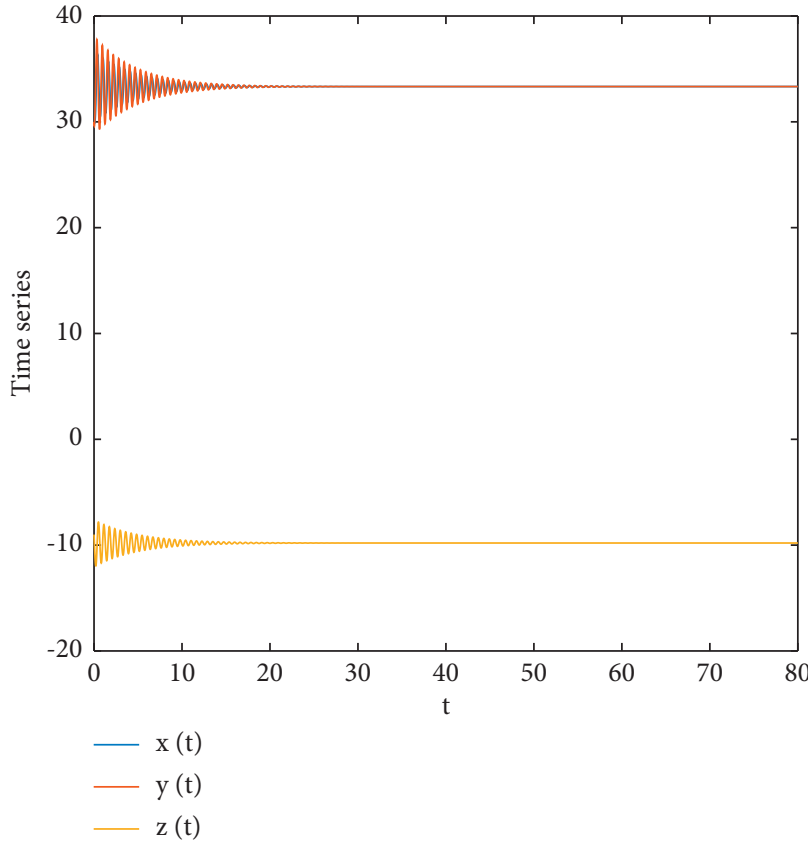

(a)

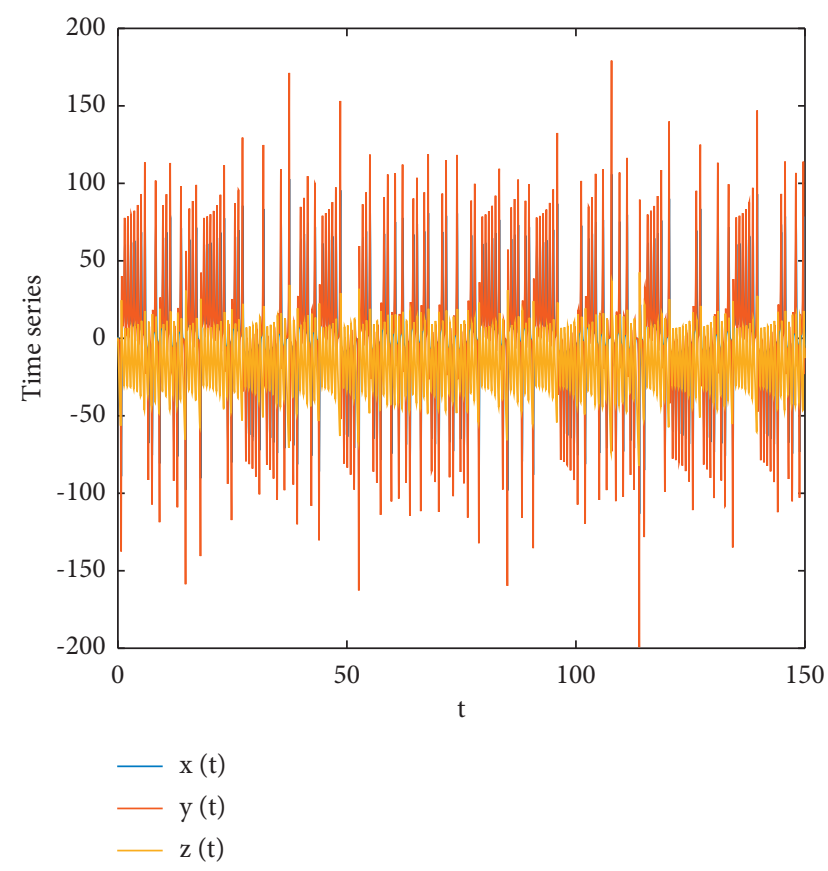

(b)

FIGURE 1: For parameter values $\left(a, b, c, k, k_{1}, k_{2}\right)=(10,100,9.8,0,0.08,0.01)$, system (1) without delay has two stable equilibria: (a) system (1) without delay tends to stable equilibrium when $\mathrm{IC}=(30,30,-9)$; (b) system (1) without delay has a chaotic attractor when $\mathrm{IC}=(0.98,-1.82,0.01)$.

(2) Otherwise, if and only if $s_{1}^{*}=(-p+\sqrt{\Delta}) / 3>0$ and $h\left(s_{1}^{*}\right) \leq 0$, equation (11) has positive roots

From the second point of Lemma 1, we can make an assumption to obtain the two positive roots of equation (11), and when $s_{1}<s_{2}$, then $h^{\prime}\left(s_{1}\right)<0$ and $h^{\prime}\left(s_{2}\right)>0$ :

$$
\begin{aligned}
\Delta & =p^{2}-3 q>0, \\
s_{1}^{*} & =\frac{-p+\sqrt{\Delta}}{3}>0, \\
h\left(s_{1}^{*}\right) & <0 .
\end{aligned}
$$

Substituting $\rho_{k}=\sqrt{k}(k=1,2)$ into equation (9), we have

$$
\tau_{k}^{j}= \begin{cases}\frac{1}{\rho_{k}}[\arccos (P)+2 j \pi], & Q \geq 0, \\ \frac{1}{\rho_{k}}[2 \pi-\arccos (P)+2 j \pi], & Q<0,\end{cases}
$$

where

$$
\begin{gathered}
P=\frac{b_{1} \rho_{k}^{2}-a_{1} b_{1}+a_{0} b_{2}-a_{2} b_{2} \rho_{k}^{2}}{b_{2}^{2} \rho_{k}^{2}+b_{1}^{2}}, \\
Q=\frac{b_{2} \rho_{k}^{4}-a_{1} b_{2} \rho_{k}^{2}+a_{2} b_{1} \rho_{k}^{2}-a_{0} b_{1}}{\rho_{k}\left(b_{2}^{2} \rho_{k}^{2}+b_{1}^{2}\right)},
\end{gathered}
$$

and $j=0,1, \ldots$. The following lemma comes naturally.
Lemma 2. If Lemma 2 holds, when $\tau=\tau_{k}^{j}(k=1,2 ; j=0,1,2, \ldots)$, the root of a system (7) consists of a number of pure imaginary roots and nonzero real parts.

By substituting $\lambda(\tau)$ into equation (7) and taking the derivative of $\tau$, we can obtain

$$
\begin{aligned}
{\left[\frac{d(\operatorname{Re} \lambda)}{d \tau}\right]_{\tau=\tau_{k}^{j}}^{-1}=} & \operatorname{Re}\left[\frac{3 \lambda^{2}+2 a_{2} \lambda+a_{1}}{\lambda\left(b_{2} \lambda^{2}+b_{1} \lambda\right) e^{-\lambda \tau}}\right]_{\tau=\tau_{k}^{j}} \\
& +\operatorname{Re}\left[\frac{2 b_{2} \lambda+b_{1}}{\lambda\left(b_{2} \lambda^{2}+b_{1} \lambda\right)}\right]_{\tau=\tau_{k}^{j}} \\
= & \operatorname{Re}\left[\frac{3 \lambda^{2}+2 a_{2} \lambda+a_{1}}{-\rho_{k}^{2}\left(b_{1}+i b_{2} \rho_{k}\right)}\left(\cos \left(\rho_{k} \tau_{k}^{j}\right)+i \sin \left(\rho_{k} \tau_{k}^{j}\right)\right)\right] \\
& +\operatorname{Re}\left[\frac{2 b_{2} \lambda+b_{1}}{-\rho_{k}^{2}\left(b_{1}+i b_{2} \rho_{k}\right)}\right] \\
= & \frac{s_{k}}{\Lambda}\left[3 \rho_{k}^{4}+2\left(a_{2}^{2}-b_{2}^{2}-2 a_{1}\right) \rho_{k}^{2}+a_{1}^{2}-2 a_{0} a_{2}-b_{1}^{2}\right] \\
= & \frac{s_{k}}{\Lambda} h^{\prime}\left(s_{k}\right),
\end{aligned}
$$

where $\Lambda=\rho_{k}^{4}\left(b_{1}^{2}+b_{2}^{2} \rho_{k}^{2}\right)$. Since $s_{k}>0$, we conclude that $[d(\operatorname{Re} \lambda) / d \tau]_{\tau=\tau^{j}}^{-1}$ and $h^{\prime}\left(s_{k}\right)$ have the same sign. Note that $h^{\prime}\left(s_{1}\right)<0$ and $h^{\prime}\left(s_{2}\right)>0$.

Thus, the following crucial lemma can be obtained. 
Lemma 3. If (15) holds, then the transversality condition of Hopf bifurcation holds: $[d(\operatorname{Re} \lambda) / d \tau]_{\tau=\tau_{1}^{j}}^{-1}<0,[d(\operatorname{Re} \lambda) / d \tau]_{\tau=\tau_{2}^{j}}^{-1}$ $>0$, where $j=0,1, \ldots$.

The results discussed above and the basic conditions for Hopf bifurcation (transversality and nondegradation) are also applicable to differential equations with time delay, and the important theorem in this section holds [33].

Theorem 1. Suppose that (6) and (15) are satisfied, then system (1) undergoes a Hopf bifurcation at the equilibria $E_{1,2}$ when $\tau=\tau_{k}^{j}(k=1,2 ; j=0,1,2, \ldots)$. Moreover, if $\tau_{1}^{0}>\tau_{2}^{0}$, then there exists $m \in N$ such that $\tau_{2}^{0}<\tau_{1}^{0}<\tau_{2}^{1}<\tau_{1}^{1}<$ $\cdots<\tau_{2}^{m}<\tau_{1}^{m}<\tau_{2}^{m+1}<\tau_{2}^{m+2}<\tau_{1}^{m+1}$ and equilibria $E_{1,2}$ of system (1) are asymptotically stable for $\tau \in\left[0, \tau_{2}^{0}\right) \bigcup\left(\tau_{1}^{0}, \tau_{2}^{1}\right)$ $\bigcup \cdots \cup\left(\tau_{1}^{m-1}, \tau_{2}^{m}\right) \bigcup\left(\tau_{1}^{m}, \tau_{2}^{m+1}\right)$ and unstable for $\tau \in\left[\tau_{2}^{0}, \tau_{1}^{0}\right)$ $\bigcup\left(\tau_{2}^{1}, \tau_{1}^{1}\right) \bigcup \cdots \bigcup\left(\tau_{2}^{m}, \tau_{1}^{m}\right) \bigcup\left(\tau_{2}^{m+1},+\infty\right)$. Furthermore, system (1) undergoes a Hopf bifurcation at the equilibria $E_{1,2}$ when $\tau=\tau_{k}^{j}(k=1,2 ; j=0,1, \ldots)$.

Remark. Theorem 3 shows that when the delay passes a certain critical value, the chaotic attractor generated by system (1) with only two stable node foci can be transformed into stable and unstable periodic orbits or another chaotic attractor. Thus, the chaos generated by system (1) is controllable.

\section{Direction and Stability of Hopf Bifurcation}

The Hopf bifurcation theory of the smooth autonomous system has been very advanced [33-35]. In this section, we use bifurcation theory to study Hopf bifurcation of system (1), determine the bifurcation direction and stability, and obtain the corresponding parameter conditions through detailed calculation. Due to the symmetry of the equilibrium point, we only study the Hopf bifurcation of $E_{1}$ at $\tau=\tau_{k}$.

Let $\quad m_{1}=x-x_{0}, m_{2}=y-y_{0}, m_{3}=z-z_{0}, \overline{m_{i}}(t)=$ $m_{i}(\tau t)$, and $\tau=\omega+\tau_{k}$; for convenience, we are dropping the bars. Nonlinear system (1) can be transformed into an FDE in $C \in C\left([-1,0], R^{3}\right)$ as

$$
\dot{m}(t)=L_{\omega}\left(m_{t}\right)+g\left(\omega, m_{t}\right),
$$

where $m(t)=\left(m_{1}(t), m_{2}(t), m_{3}(t)\right)^{T} \in R^{3}$ and $L_{\omega}: C \longrightarrow$ $R^{3}$ and $g: R \times C \longrightarrow R$ are given, respectively, by

$$
\begin{aligned}
& L_{\omega}(\phi)=\left(\omega+\tau_{k}\right)\left(\begin{array}{ccc}
-a & a & 0 \\
c & -k-c & -\frac{\sqrt{b}}{\sqrt{k_{1}+k_{2}}} \\
\frac{\sqrt{b} k_{1}}{\sqrt{k_{1}+k_{2}}} \frac{\sqrt{b}\left(k_{1}+2 k_{2}\right)}{\sqrt{k_{1}+k_{2}}} & 0
\end{array}\right)\left(\begin{array}{c}
\phi_{1}(0) \\
\phi_{2}(0) \\
\phi_{3}(0)
\end{array}\right)+\left(\omega+\tau_{k}\right)\left(\begin{array}{ccc}
0 & 0 & 0 \\
0 & k & 0 \\
0 & 0 & 0
\end{array}\right)\left(\begin{array}{l}
\phi_{1}(-1) \\
\phi_{2}(-1) \\
\phi_{3}(-1)
\end{array}\right), \\
& -\left(\omega, m_{t}\right)=\left(\omega+\tau_{k}\right)\left(\begin{array}{c}
-\phi_{1}(0) \phi_{3}(0) \\
k_{1} \phi_{1}(0) \phi_{2}(0)+k_{2} \phi_{2}^{2}(0)
\end{array}\right) .
\end{aligned}
$$

Based on the Reese representation theorem in functional analysis,

$$
L_{\omega}(\phi)=\int_{-1}^{0} d \mu(\varepsilon, \omega) \phi(\varepsilon), \quad \text { for } \phi \in C .
$$

where $\mu(\varepsilon, \omega)$ is a bounded variation function in $\varepsilon \in[-1,0]$ and can be selected as

$$
\mu(\varepsilon, \omega)=\left(\omega+\tau_{k}\right)\left(\begin{array}{ccc}
-a & a & 0 \\
c & -k-c & -\frac{\sqrt{b}}{\sqrt{k_{1}+k_{2}}} \\
\frac{\sqrt{b} k_{1}}{\sqrt{k_{1}+k_{2}}} \frac{\sqrt{b}\left(k_{1}+2 k_{2}\right)}{\sqrt{k_{1}+k_{2}}} & 0
\end{array}\right) \delta(\varepsilon)-\left(\omega+\tau_{k}\right)\left(\begin{array}{ccc}
0 & 0 & 0 \\
0 & k & 0 \\
0 & 0 & 0
\end{array}\right) \delta(\varepsilon+1),
$$


where $\delta(\cdot)$ is a Dirac function.

Let us define $A(\omega) \phi$ and $R(\omega) \phi$ on $\phi \in C\left([-1,0], R^{3}\right)$, and let $m_{t}(\varepsilon)=m(t+\varepsilon), \varepsilon \in[-1,0]$. Rewrite the system as equation (31):

$$
A(\omega) \phi=\left\{\begin{array}{l}
\frac{d \phi(\varepsilon)}{d \varepsilon}, \quad \varepsilon \in[-1,0), \\
\int_{-1}^{0} d \mu(\varepsilon, s) \phi(s), \quad \varepsilon=0,
\end{array}\right.
$$

and

$$
\begin{aligned}
R(\omega) \phi & =\left\{\begin{array}{l}
0, \quad \varepsilon \in[-1,0), \\
g(\omega, \phi), \quad \varepsilon=0,
\end{array}\right. \\
\dot{m}(t) & =A(\omega) m_{t}+R(\omega) m_{t} .
\end{aligned}
$$

For $\psi \in C^{1}\left([0,1],\left(R^{3}\right)^{*}\right)$, we can define $A^{*} \psi(\theta)$; when $\theta=0, \quad A^{*} \psi(\theta)=\int_{-1}^{0} d \mu^{T}(t, 0) \psi(-t) ; \quad$ Otherwise, when $\theta \in(0,1], A^{*} \psi(\theta)=-d \psi(\theta) / d \theta$ and we get a bilinear inner product

$$
\langle\psi, \phi\rangle=\bar{\psi}(0) \phi(0)-\int_{-1}^{0} \int_{\xi=0}^{\varepsilon} \bar{\psi}(\xi-\varepsilon) d \mu(\varepsilon) \phi(\xi) d \xi,
$$

where $\mu(\varepsilon)=\mu(\varepsilon, 0)$.

According to the properties of matrix eigenvalues, we can get that the eigenvalues of $A^{*}$ are the same as those of $A(0)$. Therefore, the eigenvalue of $A(0)$ is $\pm i \rho_{k} \tau_{k}$. We need to calculate the $i \rho_{k} \tau_{k}$ and $-i \rho_{k} \tau_{k}$ corresponding to the eigenvectors of $A(0)$ and $A^{*}$. Let $A(0) q(\varepsilon)=i \rho_{k} \tau_{k} q(\varepsilon)$, i.e., $q(\varepsilon)=e^{i \varepsilon \rho_{k} \tau_{k}}(1, \alpha, \beta)^{T}$, be the eigenvectors of $A(0)$; then, we have

$$
\left(\begin{array}{ccc}
i \rho_{k}+a & -a & 0 \\
-c & i \rho_{k}+k+c-k e^{-i \rho_{k} \tau_{k}} & \frac{\sqrt{b}}{\sqrt{k_{1}+k_{2}}} \\
-\frac{\sqrt{b} k_{1}}{\sqrt{k_{1}+k_{2}}} & -\frac{\sqrt{b}\left(k_{1}+2 k_{2}\right)}{\sqrt{k_{1}+k_{2}}} & i \rho_{k}
\end{array}\right) q(0)=\left(\begin{array}{l}
0 \\
0 \\
0
\end{array}\right) .
$$

$$
\begin{aligned}
q(0) & =(1, \alpha, \beta)^{T} \\
& =\left(1, \frac{a+i \rho_{k}}{a}, \frac{\sqrt{b}\left(-2 i a\left(k_{1}+k_{2}\right)+\left(k_{1}+2 k_{2}\right) w_{k}\right)}{a \sqrt{k_{1}+k_{2}} w_{k}}\right)^{T} .
\end{aligned}
$$

Similarly, we can assume that $q^{*}(\theta)=e^{i \theta \rho_{k} \tau_{k}} D\left(1, \alpha^{*}, \beta^{*}\right)$ is the eigenvector of $A^{*}$ corresponding to $-i \rho_{k} \tau_{k}$, that is, $A^{*} q^{*}(\theta)=-i \rho_{k} \tau_{k} q^{*}(\theta)$, and we have

$$
\begin{aligned}
q^{*}(\theta) & =D\left(1, \alpha^{*}, \beta^{*}\right) e^{i \theta \rho_{k} \tau_{k}} \\
& =D\left(1, \frac{\left(k_{1}+k_{2}\right)\left(a-i \rho_{k}\right) \rho_{k}}{-i b k_{1}+c\left(k_{1}+k_{2}\right) \rho_{k}}, \frac{\sqrt{b\left(k_{1}+k_{2}\right)}\left(a-i \rho_{k}\right)}{b k_{1}+i c\left(k_{1}+k_{2}\right) \rho_{k}}\right) e^{i \theta \rho_{k} \tau_{k}} .
\end{aligned}
$$

By (25), we can replace $\psi$ and $\phi$ with $q(0)$ and $q^{*}(\theta)$. At this time, $\left\langle q^{*}(\theta), q(\varepsilon)\right\rangle=1$, so we can calculate $D$, shown as follows:

$$
\left\langle q^{*}(\theta), q(\varepsilon)\right\rangle=\bar{D}\left\{1+\alpha \overline{\alpha^{*}}+\beta \overline{\beta^{*}}+k \tau_{k} \alpha \overline{\alpha^{*}} e^{-i \rho_{k} \tau_{k}}\right\}=1 .
$$

Therefore, we can obtain

$$
D=\frac{1}{\left\{1+\alpha \overline{\alpha^{*}}+\beta \overline{\beta^{*}}+k \tau_{k} \alpha \overline{\alpha^{*}} e^{-i \rho_{k} \tau_{k}}\right\}}
$$

The central manifold $C_{0}$ must be calculated at $\omega=0$. We can make $m_{t}$ the solution of (29) when $\omega=0$. Define

$$
z(t)=\left\langle q^{*}, m_{t}\right\rangle, G(t, \varepsilon)=m_{t}(\varepsilon)-2 \operatorname{Re}\{z(t) q(\varepsilon)\} .
$$

Then,

$$
\begin{aligned}
G(t, \varepsilon)= & G(z(t), \bar{z}(t), \varepsilon)=G_{20}(\varepsilon) \frac{z^{2}}{2}+G_{11}(\varepsilon) z \bar{z} \\
& +G_{02}(\varepsilon) \frac{\bar{z}^{2}}{2}+G_{30}(\varepsilon) \frac{z^{3}}{6}+\cdots,
\end{aligned}
$$

where $z$ and $\bar{z}$ are the local coordinates for the center manifold $C_{0}$ in the directions of $q^{*}$ and $\bar{q}^{*}$. Note that since $m_{t}$ is real, then $G$ is also real, so we only deal with real solutions. For solution $m_{t} \in C_{0}$, since $\omega=0$, we have

Through calculation, there are

$$
\begin{aligned}
\dot{z}(t) & =i \rho_{k} \tau_{k} z+\left\langle q^{*}(\varepsilon), f(0, G(z(t), t \bar{z} n(t)), \varepsilon+t 2 n \operatorname{Req}\{z(t) q(\varepsilon)\})\right\rangle \\
& =i \rho_{k} \tau_{k} z+q^{*}(0) f(0, G(z(t), \bar{z}(t), 0)+2 \operatorname{Re}\{z(t) q(0)\})
\end{aligned}
$$

Let $\quad g(0, G(z(t), \bar{z}(t), 0)+2 \operatorname{Re}\{z(t) q(0)\})=g_{0}(z, \bar{z})$; then,

$$
\dot{z}(t)=i \rho_{k} \tau_{k} z+q^{*}(0) g_{0}(z, \bar{z}) .
$$

Now, we consider

$$
\dot{z}(t)=i \rho_{k} \tau_{k} z+f(z, \bar{z}),
$$

where

$$
f(z, \bar{z})=f_{20} \frac{z^{2}}{2}+f_{11} z \bar{z}+f_{02} \frac{\bar{z}^{2}}{2}+f_{21} \frac{z^{2} \bar{z}}{2}+\cdots
$$


Since $q(\varepsilon)=(1, \alpha, \beta)^{T} e^{i \varepsilon \rho_{k} \tau_{k}} \quad$ and $\quad m_{t}(\varepsilon)=\left(m_{1 t}\right.$ $\left.(\varepsilon), m_{2 t}(\varepsilon), m_{3 t}(\varepsilon)\right)=G(t, \varepsilon)+z(t) q(\varepsilon)+\bar{z}(t) \bar{q}(\varepsilon)$, we have

$$
\begin{aligned}
& m_{1 t}(0)=z+\bar{z}+G_{20}^{(1)} \frac{z^{2}}{2}+G_{11}^{(1)} z \bar{z}+G_{02}^{(1)} \frac{\bar{z}^{2}}{2}+\cdots, \\
& m_{2 t}(0)=\alpha z+\overline{\alpha z}+G_{20}^{(2)} \frac{z^{2}}{2}+G_{11}^{(2)} z \bar{z}+G_{02}^{(2)} \frac{\bar{z}^{2}}{2}+\cdots, \\
& m_{3 t}(0)=\beta z+\bar{\beta} \bar{z}+G_{20}^{(3)} \frac{z^{2}}{2}+G_{11}^{(3)} z \bar{z}+G_{02}^{(3)} \frac{\bar{z}^{2}}{2}+\cdots
\end{aligned}
$$

$$
\begin{aligned}
f(z, \bar{z})= & q^{*}(0) g_{0}(z, \bar{z}) \\
= & \bar{D} \tau_{k}\left(1, \alpha^{*}, \beta^{*}\right)\left(\begin{array}{c}
0 \\
-m_{1 t}(0) m_{3 t}(0) \\
k_{1} m_{1 t}(0) m_{2 t}(0)+k_{2} m_{2 t}^{2}(0)
\end{array}\right) \\
= & \bar{D} \tau_{k}\left[-\alpha^{*}\left(z+\bar{z}+G_{20}^{(1)} \frac{z^{2}}{2}+G_{11}^{(1)} z \bar{z}+G_{02}^{(1)} \frac{\bar{z}^{2}}{2}+\cdots\right)\left(\beta z+\bar{\beta} \bar{z}+G_{20}^{(3)} \frac{z^{2}}{2}+G_{11}^{(3)} z \bar{z}+G_{02}^{(3)} \frac{\bar{z}^{2}}{2}+\cdots\right)\right. \\
& +k_{1} \beta^{*}\left(z+\bar{z}+G_{20}^{(1)} \frac{z^{2}}{2}+G_{11}^{(1)} z \bar{z}+G_{02}^{(1)} \frac{\bar{z}^{2}}{2}+\cdots\right)\left(\alpha z+\bar{\alpha} \bar{z}+G_{20}^{(2)} \frac{z^{2}}{2}+G_{11}^{(2)} z \bar{z}+G_{02}^{(2)} \frac{\bar{z}^{2}}{2}+\cdots\right) \\
& \left.+k_{2} \beta^{*}\left(\alpha z+\overline{\alpha z}+G_{20}^{(2)} \frac{z^{2}}{2}+G_{11}^{(2)} z \bar{z}+G_{02}^{(2)} \frac{\bar{z}^{2}}{2}+\cdots\right)^{2}\right] .
\end{aligned}
$$

$$
\begin{aligned}
f_{20}= & 2 \bar{D} \tau_{k}\left(k_{1} \alpha \overline{\beta^{*}}-\beta \overline{\alpha^{*}}+k_{2} \alpha^{2} \beta^{*}\right), \\
f_{11}= & \bar{D} \tau_{k}\left(2 k_{1} \beta^{*} \operatorname{Re}\{\alpha\}-2 \overline{\alpha^{*}} \operatorname{Re}\{\beta\}+2 k_{2} \beta^{*} \alpha \bar{\alpha}\right), \\
f_{02}= & 2 \bar{D} \tau_{k}\left(k_{1} \overline{\beta^{*}} \bar{\alpha}-\overline{\alpha^{*}} \bar{\beta}+k_{2} \bar{\alpha}^{2}\right), \\
f_{21}= & -\bar{D} \tau_{k} \alpha^{*}\left[2 G_{11}^{(3)}(0)+G_{20}^{(3)}(0)+2 \beta G_{11}^{(1)}(0)+\bar{\beta} G_{20}^{(1)}(0)\right] \\
& +k_{1} \bar{D} \tau_{k} \beta^{*}\left[2 G_{11}^{(2)}(0)+G_{20}^{(2)}(0)+2 \alpha G_{11}^{(1)}(0)+\bar{\alpha} G_{20}^{(1)}(0)\right] \\
& +2 k_{2} \bar{D} \tau_{k} \beta^{*}\left[\bar{\alpha} G_{20}^{(2)}(0)+2 \alpha G_{11}^{(2)}(0)\right] .
\end{aligned}
$$

Then, we need to compute $G_{20}(\varepsilon)$ and $G_{11}(\varepsilon)$. From (24) and (31), we have

$$
\dot{G}=\dot{m}_{t}-\dot{z} q-\dot{\bar{z}} \bar{q}=\left\{\begin{array}{l}
A(0) G-2 \operatorname{Re}\left\{\overline{q^{*}}(0) g_{0} q(\varepsilon)\right\}, \varepsilon \in[-1,0) \\
A(0) G-2 \operatorname{Re}\left\{\overline{q^{*}}(0) g_{0} q(\varepsilon)\right\}+g_{0}, \varepsilon=0 .
\end{array}\right.
$$

Let

$$
M(z, \bar{z}, \varepsilon)=\left\{\begin{array}{l}
2 \operatorname{Re}\left\{\overline{q^{*}}(0) g_{0} q(\varepsilon)\right\}, \quad \varepsilon \in[-1,0) \\
2 \operatorname{Re}\left\{\overline{q^{*}}(0) g_{0} q(\varepsilon)\right\}+g_{0}, \quad \varepsilon=0
\end{array}\right.
$$

We can rewrite (40) as

$$
\dot{G}=A(0) G+M(z, \bar{z}, \varepsilon)
$$

where

$$
M(z, \bar{z}, \varepsilon)=M_{20}(\varepsilon) \frac{z^{2}}{2}+M_{11}(\varepsilon) z \bar{z}+M_{02}(\varepsilon) \frac{\bar{z}^{2}}{2}+\cdots .
$$

According to the definition of (40) and (43) and $G$, using the series expansion and comparison of coefficients, we have $\left(A(0)-2 i \rho_{k} \tau_{k}\right) G_{20}(\varepsilon)=-M_{20}(\varepsilon), A(0) G_{11}(\varepsilon)=-M_{11}(\varepsilon), \ldots$

From (40), we know that for $\varepsilon \in[-1,0)$,

$$
\begin{aligned}
M(z, \bar{z}, \varepsilon) & =-\overline{q^{*}}(0) g_{0} q(\varepsilon)-q^{*}(0) \overline{g_{0}} \bar{q}(\varepsilon) \\
& =-f(z, \bar{z}) q(\varepsilon)-\bar{f}(z, \bar{z}) \bar{q}(\varepsilon) .
\end{aligned}
$$


Comparing the coefficients of equation (45) with those of equation (43), we have

$$
\begin{aligned}
& M_{20}(\varepsilon)=-f_{20} q(\varepsilon)-\bar{f}_{02} \bar{q}(\varepsilon), \\
& M_{11}(\varepsilon)=-f_{11} q(\varepsilon)-\bar{f}_{11} \bar{q}(\varepsilon) .
\end{aligned}
$$

Therefore, the following equation can be obtained:

$$
\dot{G_{20}}=2 i \rho_{k} \tau_{k} G_{20}(\varepsilon)+f_{20} q(\varepsilon)+\bar{f}_{02} \bar{q}(\varepsilon) .
$$

From $q(\varepsilon)=(1, \alpha, \beta)^{T} e^{i \varepsilon \omega_{k} \tau_{k}}$, we can calculate the solution of the previous equation:

$$
G_{20}(\varepsilon)=\frac{i f_{20}}{\rho_{k} \tau_{k}} q(0) e^{i \varepsilon \rho_{k} \tau_{k}}+\frac{i \bar{f}_{02}}{3 \rho_{k} \tau_{k}} \bar{q}(0) e^{-i \varepsilon \rho_{k} \tau_{k}}+W_{1} e^{2 i \varepsilon \rho_{k} \tau_{k}}
$$

and, similarly,

$$
G_{11}(\varepsilon)=\frac{i f_{11}}{\rho_{k} \tau_{k}} q(0) e^{i \varepsilon \rho_{k} \tau_{k}}+\frac{i \bar{f}_{11}}{\rho_{k} \tau_{k}} \bar{q}(0) e^{-i \varepsilon \rho_{k} \tau_{k}}+W_{2},
$$

where $\quad W_{1}=\left(W_{1}^{(1)}, W_{1}^{(2)}, W_{1}^{(3)}\right)^{T} \in R^{3} \quad$ and $W_{2}=\left(W_{2}^{(1)}, W_{2}^{(2)}, W_{2}^{(3)}\right)^{T} \in R^{3}$ are the constant vectors corresponding to the initial conditions.

We find the values of $W_{1}$ and $W_{2}$ now. For (44), we have

$$
\dot{G}_{20}(\varepsilon)=\int_{-1}^{0} d \mu(\varepsilon) G_{20}(\varepsilon)=2 i \varepsilon \rho_{k} \tau_{k} G_{20}(0)-M_{20}(0)
$$

and

$$
\dot{G}_{11}(\varepsilon)=\int_{-1}^{0} d \mu(\varepsilon) G_{11}(\varepsilon)=-M_{11}(0),
$$

where $\mu(\varepsilon)=\mu(\varepsilon, 0)$. From equation (40), we have

$$
M_{20}(0)=-f_{20} q(0)-\bar{f}_{02} \bar{q}(0)+2 \tau_{k}\left(0,-\beta, k_{1} \alpha+k_{2} \alpha^{2}\right)^{T}
$$

and

$$
\begin{aligned}
M_{11}(0)= & -f_{11} q(0)-\bar{f}_{11} \bar{q}(0) \\
& +2 \tau_{k}\left(0,-\operatorname{Re}\{\beta\}, k_{1} \operatorname{Re}\{\alpha\}+k_{2} \alpha \bar{\alpha}\right)^{T} .
\end{aligned}
$$

The eigenvector corresponding to eigenvalue $i \rho_{k} \tau_{k}$ by $A(0)$ is $q(0)$. We obtain

$$
\begin{aligned}
& \left(i \rho_{k} \tau_{k}-\int_{-1}^{0} e^{i \varepsilon \rho_{k} \tau_{k}} d \mu(f)\right) q(0) \\
& \quad=0,\left(-i \rho_{k} \tau_{k}-\int_{-1}^{0} e^{-i \varepsilon \rho_{k} \tau_{k}} d \mu(f)\right) \bar{q}(0)=0 .
\end{aligned}
$$

Substituting equations (48) and (52) into equation (50), we obtain

$$
\left(2 i \rho_{k} \tau_{k} I-\int_{-1}^{0} e^{2 i \varepsilon \rho_{k} \tau_{k}} d \mu(f)\right) E_{1}=2 \tau_{k}\left(0,-\beta, k_{1} \alpha+k_{2} \alpha^{2}\right)^{T} .
$$

That is,

$$
\begin{gathered}
\left(\begin{array}{ccc}
2 i \rho_{k}+a & -a & 0 \\
-c & 2 i \rho_{k}+k+c-k e^{-i \rho_{k} \tau_{k}} & \frac{\sqrt{b}}{\sqrt{k_{1}+k_{2}}} \\
-\frac{\sqrt{b} k_{1}}{\sqrt{k_{1}+k_{2}}} & -\frac{\sqrt{b}\left(k_{1}+2 k_{2}\right)}{\sqrt{k_{1}+k_{2}}} & 2 i \rho_{k}
\end{array}\right) E_{1} \\
\left.\quad \begin{array}{c}
0 \\
-\beta \\
k_{1} \alpha+k_{2} \alpha^{2}
\end{array}\right) .
\end{gathered}
$$

It follows that

$$
\begin{aligned}
& W_{1}^{(1)}=\frac{\Delta_{11}}{\Delta_{1}}, \\
& W_{1}^{(2)}=\frac{\Delta_{12}}{\Delta_{1}},
\end{aligned}
$$

$$
W_{1}^{(3)}=\frac{\Delta_{13}}{\Delta_{1}} \text {, }
$$

where 


$$
\Delta_{11}=2\left|\begin{array}{ccc}
0 & -a & 0 \\
-\beta & 2 i \rho_{k}+k+c-k e^{-i \rho_{k} \tau_{k}} & \frac{\sqrt{b}}{\sqrt{k_{1}+k_{2}}} \\
k_{1} \alpha+k_{2} \alpha^{2} & -\frac{\sqrt{b}\left(k_{1}+2 k_{2}\right)}{\sqrt{k_{1}+k_{2}}} & 2 i \rho_{k}
\end{array}\right|, \quad W_{2}^{(2)}=\frac{\Delta_{22}}{\Delta_{2}},
$$$$
\Delta_{12}=2\left|\begin{array}{ccc}
2 i \rho_{k}+a & 0 & 0 \\
-c & -\beta & \frac{\sqrt{b}}{\sqrt{k_{1}+k_{2}}} \\
-\frac{\sqrt{b} k_{1}}{\sqrt{k_{1}+k_{2}}} & k_{1} \alpha+k_{2} \alpha^{2} & 2 i \rho_{k}
\end{array}\right|,
$$$$
\Delta_{13}=2\left|\begin{array}{ccc}
2 i \rho_{k}+a & -a & 0 \\
-c & 2 i \rho_{k}+k+c-k e^{-i \rho_{k} \tau_{k}} & -\beta \\
-\frac{\sqrt{b} k_{1}}{\sqrt{k_{1}+k_{2}}} & -\frac{\sqrt{b}\left(k_{1}+2 k_{2}\right)}{\sqrt{k_{1}+k_{2}}} & k_{1} \alpha+k_{2} \alpha^{2}
\end{array}\right|,
$$$$
\Delta_{1}=\left|\begin{array}{ccc}
2 i \rho_{k}+a & -a & 0 \\
-c & 2 i \rho_{k}+k+c-k e^{-i \rho_{k} \tau_{k}} & \frac{\sqrt{b}}{\sqrt{k_{1}+k_{2}}} \\
-\frac{\sqrt{b} k_{1}}{\sqrt{k_{1}+k_{2}}} & -\frac{\sqrt{b}\left(k_{1}+2 k_{2}\right)}{\sqrt{k_{1}+k_{2}}} & 2 i \rho_{k}
\end{array}\right| .
$$

Similarly, substituting equations (49) and (53) into equation (51), we have

$$
\begin{gathered}
\left(\begin{array}{ccc}
a & -a & 0 \\
-c & c & \frac{\sqrt{b}}{\sqrt{k_{1}+k_{2}}} \\
-\frac{\sqrt{b} k_{1}}{\sqrt{k_{1}+k_{2}}} & -\frac{\sqrt{b}\left(k_{1}+2 k_{2}\right)}{\sqrt{k_{1}+k_{2}}} & 0
\end{array}\right) E_{2} \\
=2\left(\begin{array}{c}
0 \\
-\operatorname{Re}\{\beta\} \\
k_{1} \operatorname{Re}\{\alpha\}+k_{2} \alpha \bar{\alpha}
\end{array}\right) .
\end{gathered}
$$

It follows that where

$$
\Delta_{21}=2\left|\begin{array}{ccc}
0 & -a & 0 \\
-\operatorname{Re}\{\beta\} & c & \frac{\sqrt{b}}{\sqrt{k_{1}+k_{2}}} \\
k_{1} \operatorname{Re}\{\alpha\}+k_{2} \alpha \bar{\alpha} & -\frac{\sqrt{b}\left(k_{1}+2 k_{2}\right)}{\sqrt{k_{1}+k_{2}}} & 0
\end{array}\right|,
$$$$
\Delta_{22}=2\left|\begin{array}{ccc}
a & 0 & 0 \\
-c & -\operatorname{Re}\{\beta\} & \frac{\sqrt{b}}{\sqrt{k_{1}+k_{2}}} \\
-\frac{\sqrt{b} k_{1}}{\sqrt{k_{1}+k_{2}}} & k_{1} \operatorname{Re}\{\alpha\}+k_{2} \alpha \bar{\alpha} & 0
\end{array}\right|,
$$$$
\Delta_{23}=2\left|\begin{array}{ccc}
a & -a & 0 \\
-c & c & -\operatorname{Re}\{\beta\} \\
-\frac{\sqrt{b} k_{1}}{\sqrt{k_{1}+k_{2}}} & -\frac{\sqrt{b}\left(k_{1}+2 k_{2}\right)}{\sqrt{k_{1}+k_{2}}} & k_{1} \operatorname{Re}\{\alpha\}+k_{2} \alpha \bar{\alpha}
\end{array}\right|,
$$

$$
\Delta_{2}=\left|\begin{array}{ccc}
a & -a & 0 \\
-c & c & \frac{\sqrt{b}}{\sqrt{k_{1}+k_{2}}} \\
-\frac{\sqrt{b} k_{1}}{\sqrt{k_{1}+k_{2}}} & -\frac{\sqrt{b}\left(k_{1}+2 k_{2}\right)}{\sqrt{k_{1}+k_{2}}} & 0
\end{array}\right| .
$$

We can determine $G_{20}(0)$ and $G_{11}(0)$, and therefore, all $f_{i j}$ can be determined by (39).

In summary, the properties of Hopf bifurcation are determined by the following parameters: $\omega_{2}$ determines the direction of Hopf bifurcation, $\beta_{2}$ determines the stability of bifurcation periodic solutions, and $T_{2}$ determines the period of bifurcation periodic solutions, and the specific values are shown as follows. The main theories and methods are from $[34,35]$ : 


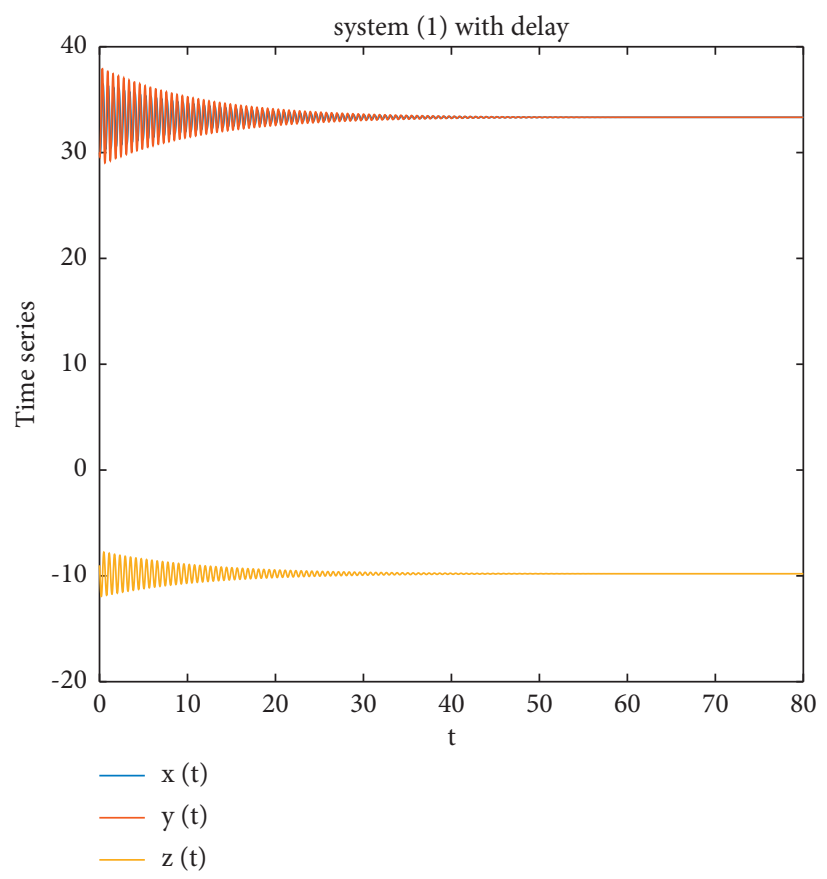

FIgURe 2: For system (1) with parameter values $\left(a, b, c, k, k_{1}, k_{2}\right)=(10,100,9.8,-0.5,0.08,0.01)$ and initial values $(30,30,-9)$ when $\tau=0.16$, the equilibrium $E_{1}$ is asymptotically stable.

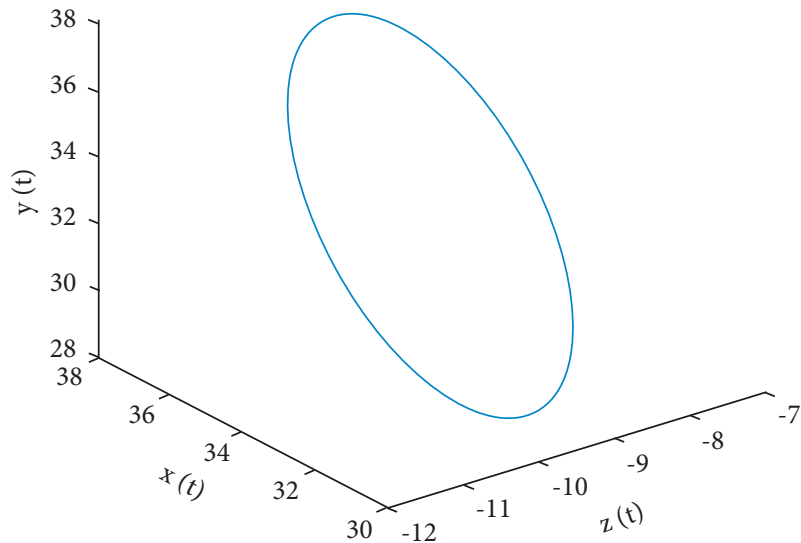

(a)

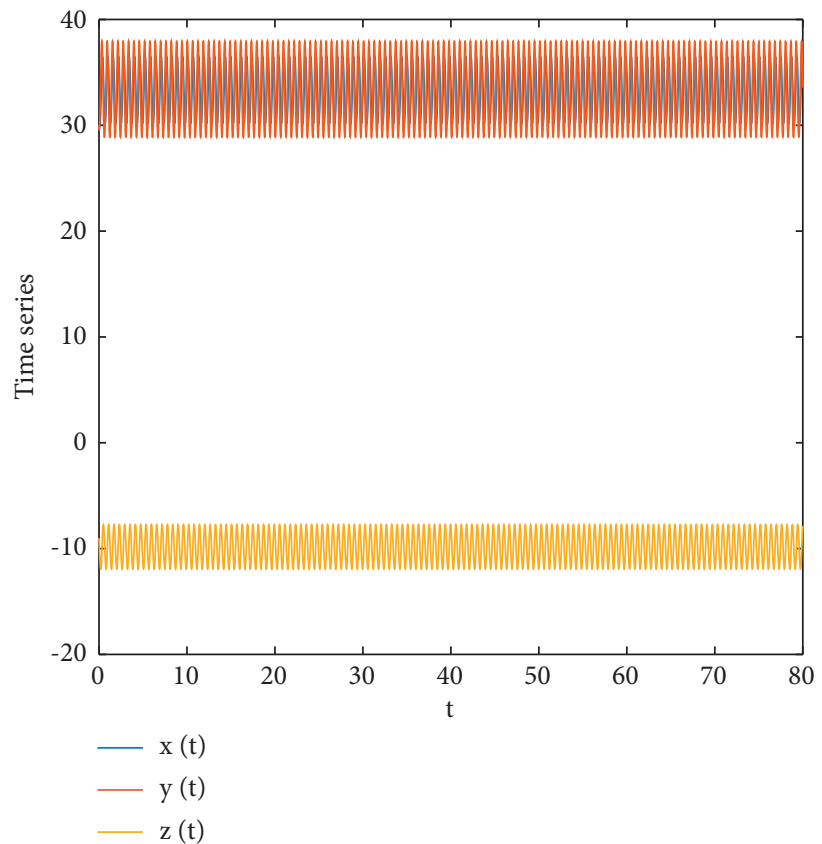

(b)

Figure 3: A bifurcation diagram for system (1) with parameter values $\left(a, b, c, k, k_{1}, k_{2}\right)=(10,100,9.8,-0.5,0.08,0.01)$ and initial values $(30,30,-9)$ when $\tau=0.2095<\tau_{2}^{0}$ is close to $\tau_{2}^{0}$ : (a) phase portrait; (b) time series of $z(t)$. 


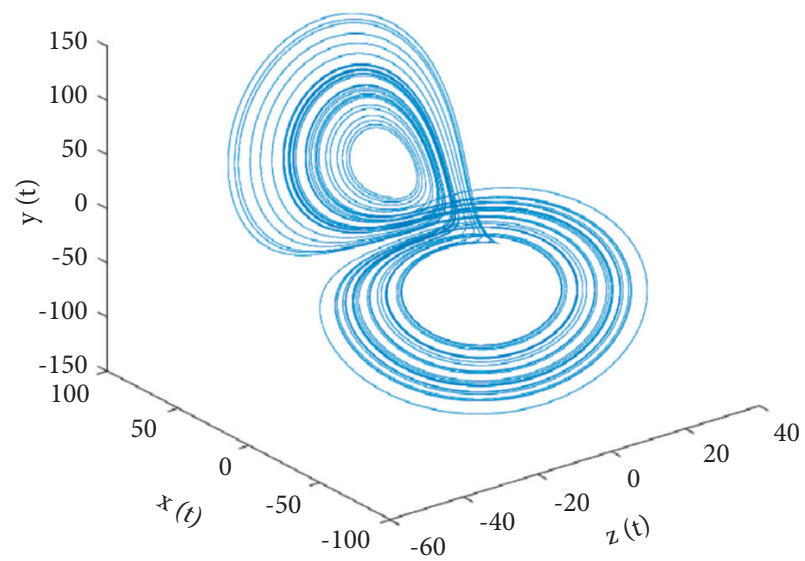

(a)

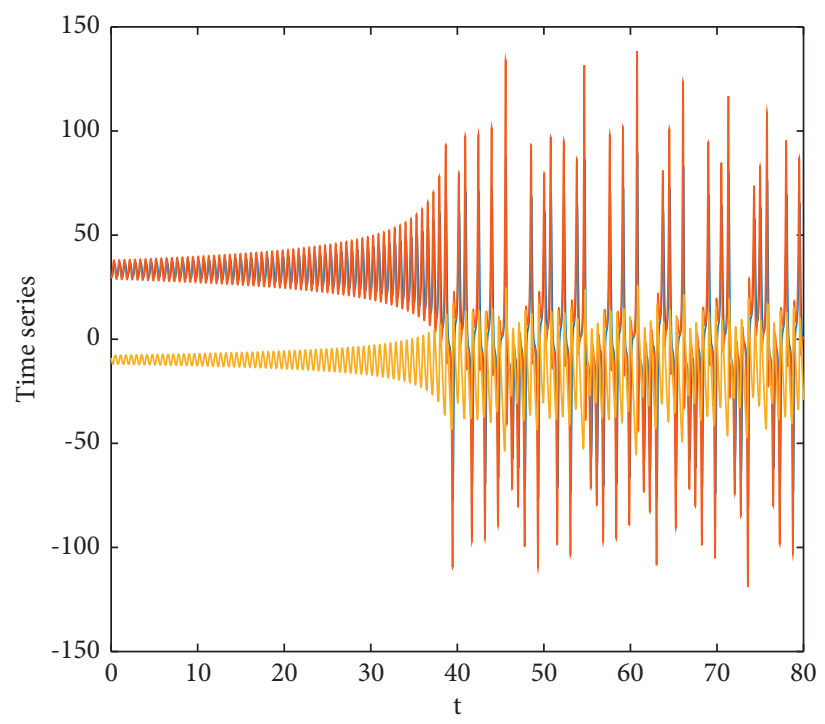

(b)

Figure 4: When $\tau$ is closer to $\tau_{2}^{0}, \tau=0.23>\tau_{2}^{0}$ is taken and system (1) is chaotic at the initial values $(30,30,-9)$ : (a) the chaotic attractor of system (1); (b) the time series of $z(t)$.

$$
\begin{aligned}
C_{1}(0) & =\frac{i}{2 \rho_{k} \tau_{k}}\left(f_{20} f_{11}-2\left|f_{11}\right|^{2}-\frac{1}{3}\left|f_{02}\right|^{2}\right)+\frac{f_{21}}{2}, \\
\omega_{2} & =-\frac{\operatorname{Re}\left\{C_{1}(0)\right\}}{\operatorname{Re}\left\{d \lambda\left(\tau_{k}\right) / d \tau\right\}}, \\
T_{2} & =-\frac{\operatorname{Im} C_{1}(0)+\omega_{2} \operatorname{Im}\left\{d \lambda\left(\tau_{k}\right) / d \tau\right\}}{\rho_{k} \tau_{k}}, \\
\beta_{2} & =2 \operatorname{Re}\left\{C_{1}(0)\right\} .
\end{aligned}
$$

Therefore, the following main results are obtained in this section.

Theorem 2. In equation (62), when $\tau>\tau_{k}$ or $\tau<\tau_{k}$, system (1) has Hopf bifurcations with the following properties: if $\omega_{2}>0\left(\omega_{2}<0\right)$, the Hopf bifurcation is supercritical (subcritical); if $\beta_{2}<0\left(\beta_{2}>0\right)$, the orbit is stable (unstable); if $T_{2}>0\left(T_{2}<0\right)$, then the period increases (decreases).

\section{Numerical Results}

In the previous two sections, we have proved the parameter conditions for Hopf bifurcation in system (1) and analyzed the bifurcation direction and bifurcation stability. In this section, we select appropriate parameters and use the MATLAB toolkit for numerical simulation to verify our theoretical analysis. When the parameter values are $a=10, c=9.8$, and $b=100$, the two equilibria are $E_{1}(100 / 3,100 / 3,-9.8)$ and $E_{2}(-100 / 3,-100 / 3,-9.8)$.

$$
\left\{\begin{array}{l}
\dot{x}=10(y-x) \\
\dot{y}=-9.8 y-x z+k[y(t-\tau)-y] \\
\dot{z}=-100+x y
\end{array}\right.
$$

Through the previous analysis and numerical simulation from the finite difference method (FDM), as shown in Figure 1 , the equilibrium points $E_{1,2}$ of system (63) are asymptotically stable when $\tau=0$ and a chaotic attractor appears. If we choose the parameter $k=-0.5$, we can get that equation (10) has two positive roots $\rho_{1}=10.0521$ and $\rho_{2}=10.3648$. Therefore, there are, respectively,

$$
\begin{aligned}
& \tau_{1}^{j}=0.467983+0.625063 j, \\
& \tau_{2}^{j}=0.211299+0.606205 j,
\end{aligned}
$$

where $j=0,1,2, \ldots$. From formula (62), it follows that $\tau_{2}^{0}=0.211299, C_{1}(0)=0.000588642+0.000658409 i, \omega_{2}=$ $-0.000362443, T_{2}=-0.000204142$, and $\beta_{2}=0.00117728$.

Therefore, as shown by computer simulation, when $0<\tau<\tau_{2}^{0}$, the equilibria $E_{1,2}$ are stable (see Figure 2). $E_{1}$ loses its stability and Hopf bifurcation occurs as $\tau$ crosses the critical value $\tau_{2}^{0}$. According to the properties of Hopf bifurcation, Hopf bifurcation is subcritical and the bifurcation direction is $\tau<\tau_{2}^{0}$, when $\omega_{2}<0$ and $\beta_{2}>0$. At this time, an unstable bifurcation periodic solution appears, as shown in Figures 3(a) and 3(b).

Otherwise, as shown in Figures 4(a) and 4(b), numerical simulation shows that when $\tau$ reaches the region $\tau>\tau_{2}^{0}$, Hopf bifurcation periodic solution disappears and chaos occurs.

\section{Conclusion}

In previous studies, few scholars have analyzed the timedelay feedback chaotic system with two stable node foci coexisting. In this paper, a unified chaotic system control model is established by using the delay feedback control law. The corresponding parameter range is obtained according to the conditions of Hopf bifurcation. Central manifold theory and normal form method are the most classical methods to 
study the properties of Hopf bifurcation. This paper also uses this method to study the direction of Hopf bifurcation and the stability of bifurcation periodic solution of system (1). Theoretical results and numerical simulation show that chaos can be controlled using a delay system (1). Numerical simulation shows that the periodic solution is transformed into a chaotic attractor with further increase in delay. It is worth noting that the results obtained in this paper are of great significance for controlling chaos in systems with only two stable node foci. The dynamic behavior of the new system is still rich and complex, and its topology needs to be thoroughly studied and developed. In future studies, we will provide more credible theoretical analysis and data results.

\section{Data Availability}

The data that support the findings of this study are included within the article.

\section{Conflicts of Interest}

The authors declare that there are no conflicts of interest regarding the publication of this paper.

\section{Acknowledgments}

This work was supported by Yulin Normal University of Scientific Research Fund for High-Level Talents (No. G2021ZK06).

\section{References}

[1] E. N. Lorenz, "Deterministic nonperiodic flow," Journal of the Atmospheric Sciences, vol. 20, no. 2, pp. 130-141, 1963.

[2] J. C. Sprott, "Some simple chaotic flows," Physical Review. E, Statistical Physics, Plasmas, Fluids, and Related Interdisciplinary Topics, vol. 50, pp. R647-R650, 1994.

[3] J. C. Sprott, "A new class of chaotic circuit," Physics Letters A, vol. 266, no. 1, pp. 19-23, 2000.

[4] J. C. Sprott, "Simplest dissipative chaotic flow," Physics Letters $A$, vol. 228, no. 4-5, pp. 271-274, 1997.

[5] G. R. Chen G and T. Ueta, "Yet another chaotic attractor," International Journal of Bifurcation and Chaos, vol. 09, no. 7, pp. 1465-1466, 1999.

[6] G. van der Schrier and L. R. M. Maas, "The diffusionless Lorenz equations; Shil'nikov bifurcations and reduction to an explicit map," Physica D: Nonlinear Phenomena, vol. 141, no. 1-2, pp. 19-36, 2000.

[7] Q. Yang and G. Chen, "A chaotic system with one saddle and two stable node-foci," International Journal of Bifurcation and Chaos, vol. 18, no. 5, pp. 1393-1414, 2008.

[8] C. Sparrow, The Lorenz Equations: Bifurcation, Chaos, and Strange Attractor, Springer-Verlag, New York, NY, USA, 1982.

[9] T. Zhou, Y. Tang, and G. Chen, "Complex dynamical behaviors of the chaotic chen's system," International Journal of Bifurcation and Chaos, vol. 13, no. 9, pp. 2561-2574, 2003.

[10] Q. Yang, G. Chen, and T. Zhou, "A unified Lorenz-type system and its canonical form," International Journal of Bifurcation and Chaos, vol. 16, no. 10, pp. 2855-2871, 2006.

[11] H. Kokubu and R. Roussarie, "Existence of a singularly degenerate heteroclinic cycle in the lorenz system and its dynamical consequences: Part I," Journal of Dynamics and Differential Equations, vol. 16, no. 2, pp. 513-557, 2004.

[12] M. Messias, "Dynamics at infinity and the existence of singularly degenerate heteroclinic cycles in the Lorenz system," Journal of Physics A: Mathematical and Theoretical, vol. 42, no. 11, Article ID 115101, 2009.

[13] L. F. Mello and S. F. Coelho, "Degenerate Hopf bifurcations in the Lü system," Physics Letters A, vol. 373, no. 12-13, pp. 1116-1120, 2009.

[14] J. Li and J. Zhang, "New treatment on bifurcations of periodic solutions and homoclinic orbits at high $\mathrm{r}$ in the lorenz equations," SIAM Journal on Applied Mathematics, vol. 53, no. 4, pp. 1059-1071, 1993.

[15] D. Huang, "Periodic orbits and homoclinic orbits of the diffusionless Lorenz equations," Physics Letters A, vol. 309, no. 3-4, pp. 248-253, 2003.

[16] Z. Wei and Q. Yang, "Controlling the diffusionless Lorenz equations with periodic parametric perturbation," Computers and Mathematics with Applications, vol. 58, no. 10, pp. 1979-1987, 2009.

[17] I. Pehlivan and Y. Uyaroglu, "A new chaotic attractor from general lorenz system family and its electronic experimental implementation," Turkish Journal of Electrical Engineering and Computer Sciences, vol. 18, pp. 171-184, 2010.

[18] Z. Wang, "Existence of attractor and control of a 3D differential system," Nonlinear Dynamics, vol. 60, no. 3, pp. 369-373, 2009.

[19] Z. Wei, B. Zhu, J. Yang, M. Perc, and M. Slavinec, "Bifurcation analysis of two disc dynamos with viscous friction and multiple time delays," Applied Mathematics and Computation, vol. 347, pp. 265-281, 2019.

[20] Q. Yang, Z. Wei, and G. Chen, “An unusual 3D autonomous quadratic chaotic system with two stable node-foci," International Journal of Bifurcation and Chaos, vol. 20, no. 04, pp. 1061-1083, 2010.

[21] Z. Wei and Q. Yang, "Dynamical analysis of a new autonomous 3-D chaotic system only with stable equilibria," Nonlinear Analysis: Real World Applications, vol. 12, no. 1, pp. 106-118, 2011.

[22] Z. Wei and Q. Yang, "Anti-control of Hopf bifurcation in the new chaotic system with two stable node-foci," Applied Mathematics and Computation, vol. 217, no. 1, pp. 422-429, 2010.

[23] Z. C. Wei, W. Zhang, I. Moroz, and N. V. Kuznetsov, "Codimension one and two bifurcations in Cattaneo Christov heat-flux model," Discrete and Continuous Dynamical Systems-Series B, vol. 26, no. 10, pp. 5305-5319, 2021.

[24] Z. Wang, F. Parastesh, K. Rajagopal, I. I. Hamarash, and I. Hussain, "Delay-induced synchronization in two coupled chaotic memristive Hopfield neural networks," Chaos, Solitons \& Fractals, vol. 134, Article ID 109702, 2020.

[25] G. A. Leonov, N. V. Kuznetsov, and T. N. Mokaev, "Hidden attractor and homoclinic orbit in Lorenz-like system describing convective fluid motion in rotating cavity," Communications in Nonlinear Science and Numerical Simulation, vol. 28, no. 1-3, pp. 166-174, 2015.

[26] M.-F. Danca and M. Lampart, "Hidden and selfexcited attractors in a heterogeneous Cournot oligopoly model," Chaos, Solitons \& Fractals, vol. 142, Article ID 110371, 2021.

[27] N. Wang, G. Zhang, N. V. Kuznetsov, and H. Bao, "Hidden attractors and multistability in a modified Chua's circuit," Communications in Nonlinear Science and Numerical Simulation, vol. 92, Article ID 105494, 2021. 
[28] K. Pyragas and A. Tamaševičius, "Experimental control of chaos by delayed self-controlling feedback," Physics Letters A, vol. 180, no. 1-2, pp. 99-102, 1993.

[29] Z. Wei, A. Yousefpour, H. Jahanshahi, U. Erkin Kocamaz, and I. Moroz, "Hopf bifurcation and synchronization of a fivedimensional self-exciting homopolar disc dynamo using a new fuzzy disturbance-observer-based terminal sliding mode control," Journal of the Franklin Institute, vol. 358, no. 1, pp. 814-833, 2021.

[30] Z. Wang, X. Xi, L. Kong, and Z. Wei, "Infinity dynamics and DDF control for a chaotic system with one stable equilibrium," The European Physical Journal-Special Topics, vol. 229, no. 6, pp. 1319-1333, 2020.

[31] Z. Wang, W. Sun, Z. Wei, and S. Zhang, "Dynamics and delayed feedback control for a 3D jerk system with hidden attractor," Nonlinear Dynamics, vol. 82, no. 1, pp. 577-588, 2015.

[32] C. Xu, Z. Liu, M. Liao, P. Li, Q. Xiao, and S. Yuan, "Fractionalorder bidirectional associate memory (BAM) neural networks with multiple delays: the case of Hopf bifurcation," Mathematics and Computers in Simulation, vol. 182, pp. 471-494, 2021.

[33] J. Hale, Theory of Functional Differential Equations, Springer, New York, NY, USA, 1977.

[34] B. Hassard, N. Kazarinoff, and Y. Wan, Theory and Application of Hopf Bifurcation, Cambridge University Press, Cambridge, UK, 1981.

[35] Y. Song and J. Wei, "Bifurcation analysis for Chen's system with delayed feedback and its application to control of chaosws," Chaos, Solitons and Fractals, vol. 22, no. 1, pp. 75-91, 2004. 